\title{
Separating comprehension from the verification process
}

\author{
IN-MAO LIU \\ National Taiwan University, Taipei, Taiwan, Republic of China
}

\begin{abstract}
The present experiments aimed at separating comprehension of a sentence from its verification. Presentation of a first sentence was terminated by the subject's keypress after his comprehension. This gave a measure of sentence comprehension. A second sentence appeared immediately for a fixed interval. Then, a picture was presented, and the subject verified the first sentence against the picture. Finally, a second picture appeared, and the subject verified the second sentence against the second picture. The purpose of presenting a second sentence was to stop the processing of the first sentence after the subject's keypress (so that comprehension did not continue beyond what was measured) and also to insure the subject's full comprehension (comprehending the sentence to form a unitary representation) that would resist forgetting in the face of processing a second sentence. The purpose of presenting a second picture was to insure the subject's processing of a second sentence. Using this experimental technique, an atypical pattern of verification latencies (TA $<$ FA $<\mathrm{TN}<\mathrm{FN}$ ) was obtained (Experiment 1). When a sentence could be falsified in more than one way (Experiment 2), TN was found to be about equal to FN. A theoretical model was proposed to account for the present and the previous findings.
\end{abstract}

In a typical sentence-picture verification task, a sentence (e.g., "The dots are red" or "The dots aren't red") and a picture (an array of dots, all of which are either red or green) are shown. The subject is timed while he reads the sentence, looks at the array, and decides whether it is true or false of the sentence. On various trials, therefore, the sentence can be affirmative or negative and either true or false. The two main results with this task are as follows. First, negative sentences take longer to verify. Second, there is an interaction between the kind of sentence (affirmative or negative) and the type of response (true or false). True affirmatives (TAs) are verified faster than false affirmatives (FAs), but false negatives (FNs) are verified faster than true negatives (TNs). Both of these results (summarized as TA $<$ FA $<$ FN $<$ TN) were obtained by a number of studies (e.g., Clark \& Chase, 1972; Just \& Carpenter, 1971).

Many theoretical accounts (Carpenter \& Just, 1975; Clark \& Chase, 1972; Gough, 1965; Trabasso, Rollins, \& Shaughnessy, 1971) of these two results have one feature in common: an "inside-out" comparison of sentence and picture representations. Mainly, the propositional encodings of the sentence and the picture are compared beginning with the innermost embedded predicates. The verification time for FN is

This study was supported by National Science Council Grant 68H-03-02(02), Republic of China. The author is grateful to May-jane Chen for commenting on the study and to Guangying Wei and Chao-ying Wang for their assistance. Address correspondence to In-mao Liu, Department of Psychology, National Taiwan University, Taipei, Taiwan. shorter than for TN because the predicates in the FN and picture representations match, while those in the TN and picture mismatch.

This theoretical model that is based on the notion of constituent-by-constituent comparisons seems at odds with another line of research (e.g., Bransford, Barclay, \& Franks, 1972; Fillenbaum, 1966; Sachs, 1967). These investigators found that, after comprehending sentence or prose materials, the subjects are likely to remember the gist of what was said rather than the exact sentence constituents. According to this finding, the subjects tend to confuse, in a recognition test, sentences that have the same meaning. In a sentence-picture verification task, the subjects should then rely mainly on the overall meaning or some unitary representation of comprehended sentences in making judgments.

It is therefore inevitable that there have been somewhat bizarre observations from the viewpoint of the constituent comparison model. Two such observations are as follows: One is obtained when the subjects fail to comprehend sentences by adopting the constituent-by-constituent comparison strategy and yet correctly verify the sentences. The other is obtained when the subjects are placed in a situation to comprehend a sentence as unitary. Let us consider these observations in some detail.

In a study by Liu and Liang (1977), the sentence for verification was of the form, "The figure is (isn't) a red triangle." It was presented simultaneously with a picture of a red triangle (true), a red rectangle (false), a green triangle (false), or a green rectangle (false). A novelty in this experiment was that a 
picture of an object was described by two words (color and shape). Had the subject comprehended a red triangle as a single entity, the same pattern of verification times would have been obtained as in the case of sentences describing a single-attribute object. A problem with this situation is that there are three types of TN. Thus, "The figure isn't a red triangle" is a TN when a picture of a red rectangle or a green triangle as well as a picture of a green rectangle is presented for verification. It was found that the three types of TN were no longer always verified slower than FN. With negatives, it was as if the subject was looking for an attribute of mismatch, resulting in a longer mean verification time for $\mathrm{FN}$ because it has no attribute that mismatches. This model of constituent-by-constituent comparisons can be refined to account for the verification of sentences describing three-attribute objects and other complicated sentences such as "This figure is red or (and) triangular" (Liang \& Liu, 1977). With the latter, for instance, the pattern of 16 mean verification times can be predicted by noting whether the connective is "or" or "and," whether the sentence is affirmative or negative, and, most important, whether a match or a mismatch is to be detected. In case a match is to be detected, the subject presses a "true" or a "false" key as soon as a match is detected without reading the remaining sentence elements. In other words, the subject correctly verifies a sentence against a picture without understanding the meaning of the sentence. This is the first type of bizarre observation that is unexpected by the constituent comparison model. It is accommodated by the constituent comparison model only at great cost.

There are several conditions in which the subject tends to comprehend even a negative sentence as unitary. In these conditions, the FN $<\mathrm{TN}$ ordering predicted by the constituent comparison model was frequently not obtained. Instead of visual presentations of sentences, when sentences were read to the subjects, Gough $(1965,1966$, Experiment 1) found that the mean verification time of $\mathrm{TN}$ was not different from that of FN. In the case of auditory presentations, apparently the subjects tended to perceive the entire meaning of a sentence. When a sufficient amount of time is allowed for the subject to process a negative sentence, he may adopt a strategy of recoding negative sentences into their affirmative counterparts in order to facilitate a later verification test. Thus, in the Carpenter and Just (1975) study, a sentence appeared in a tachistoscope for $2 \mathrm{sec}$ and then disappeared as a picture was presented for a verification test. In this situation, TNs were verified faster than FNs for Blocks 2-7 of their Experiment 2. The same reverse ordering of $F N$ and $T N$ verification times was also obtained by Trabasso et al. (1971). These investigators measured the comprehension and verification times separately by presenting sentences first. The subject pressed a key to indicate his comprehension of the sentence, and a picture followed immediately. The subject then verified the sentence against the picture. Recently, McLeod, Hunt, and Mathews (1978) followed the procedure of Trabasso et al. in which the comprehension time of a sentence was subject controlled. They isolated two groups of subjects on the basis of whether the verification times of individual subjects were fit to the Carpenter and Just constituent comparison model. The larger group was well fit, whereas the smaller group was poorly fit by the model with the TN < FN ordering obtained. Moreover, the mean comprehension time of the smaller group was found to be about $1,000 \mathrm{msec}$ longer than that of the larger group, indicating that the subjects of the smaller group were taking advantage of the subject-controlled comprehension time by using extra time to recode negative sentences into affirmatives, in order to be ready for a subsequent verification task. In a single experiment, Glushko and Cooper (1978) manipulated the amount of time of presenting a sentence alone (preparation time) before a picture was presented for a verification test. In an extreme condition, a sentence and a picture were presented simultaneously, as in most studies of sentence-picture verification. In another extreme condition, the preparation time was subject controlled, as in the study of Trabasso et al. (1971). In between these two extreme conditions, there were 6- and 2-sec deadline conditions. Although Glushko and Cooper did not use negative sentences, they found that as the preparation time was made more available to the subjects, the sentence complexity (one vs. two lines of sentences) effects disappeared gradually in their verification times. If the more complex sentences of the Glushko and Cooper study are identified with negative sentences, then the finding of Glushko and Cooper is in line with the observations of Carpenter and Just (1975), Gough (1965, 1966), McLeod et al. (1978), and Trabasso et al. (1971).

As the foregoing two paragraphs reveal, the first type of bizarre observation may easily be eliminated if the subject is given enough time to comprehend a sentence before presenting a picture for verification. There is difficulty with respect to the second type of observation, because the processing of a sentence presented during the preparation time may persist beyond this interval and continue at the time of verification. As the pattern of verification times is dependent on the length of preparation time (Glushko \& Cooper, 1978), it becomes more important to separate preparation and verification times unambiguously.

A clear separation of comprehension and verification processes may be accomplished by adopting a masking procedure, as follows: (1) A sentence 
(Sentence 1; e.g., These dots are red) is presented for comprehension, and its presentation interval is controlled by the subject. (2) Another sentence (Sentence 2; e.g., Car hits tree) is then presented for a fixed interval to stop the processing of Sentence 1 . (3) A picture (Picture 1; e.g., a picture of red dots) is presented for the verification of Sentence 1. And (4) another picture (Picture 2; e.g., a picture of a car hitting a tree) is presented for the verification of Sentence 2. The procedure then consists of four successive events.

The critical point in this procedure is the presentation of Sentence 2 and its verification. Several remarks are in order. First, this procedure guarantees the subject's full comprehension of Sentence 1. By the full comprehension of a simple sentence, we mean that the subject finds a relationship among all elements within the sentence and constructs a unitary representation. Since a retention interval (Event 2) is inserted between the comprehension (Event 1) and verification (Event 3) processes unless the subject succeeds in building a unitary representation, a string of words constituting Sentence 1 should be quickly lost from short-term memory. Then, the subject would be unable to verify Sentence 1 against Picture 1. Second, this procedure guarantees a complete separation of the comprehension and verification processes of Sentence 1, if the presentation interval of Sentence 2 is properly adjusted. If this interval is too long, the subject may continue processing Sentence 1 during Event 2 without affecting the comprehension of Sentence 2. This interval should be set in such a way that the error rate of Sentence 2 is larger than that of Sentence 1 and yet less than $50 \%$. The former condition states that the subject is overloaded at the time of processing Sentence 2 and hence not likely to continue processing Sentence 1. The latter condition states that the subject is actually processing Sentence 2 with a better-than-chance performance level.

\section{EXPERIMENT 1}

\footnotetext{
Method

Subjects and Materials. The subjects were 29 freshmen of an introductory psychology class. They were not paid.

When translated word for word from Chinese to English, Sentence I was "These dots are (not are) red" or "These dots are (not are) green." Sentences used as Sentence 2 had no word in common with Sentence 1. They were "Car hit (not hit) tree" and "Car catch up (not catch up) truck." A correct translation of the last set of sentences should be "A car has (hasn't) caught up a truck," since "catch up" and "has caught up" are indistinguishable in Chinese. Except that a negative marker generally precedes a verb, the word order in Chinese is about the same as in English. There were two pictures for verifying Sentence 1. They were either a picture of six red dots or a picture of six green dots. There were four pictures for verifying
}

Sentence 2: pictures of a car having caught up with a truck (running in parallel), of a car behind a truck, of a car hitting a tree, and of a car not hitting a tree (a car passing by a tree).

Design and Procedure. With Sentences 1 and 2, four each, and two pictures for verifying Sentence I, there were $4 \times 4 \times 2=32$ arrangements. For each arrangement a picture for verifying Sentence 2 was included in such a way that there were equal numbers of TA, FA, TN, and FN not only for Sentence 1, but also for Sentence 2

An example of a trial, then, consists of the following sequence of events. The purpose of each event is indicated in parentheses. (1) THESE DOTS ARE RED (for measuring the comprehension time). (2) CAR HITS TREE (presented for $800 \mathrm{msec}$ to stop the processing of Sentence 1). (3) A picture of red dots (for verifying Sentence 1). (4) A picture of a car hitting a tree (for verifying Sentence 2).

Approximately $2 \mathrm{sec}$ after the experimenter's ready signal, Sentence 1 was presented on the first window of a tachistoscope, and a timer started. When the subject comprehended the sentence, he pressed a key to stop the timer. Sentence 1 disappeared at that time, and Sentence 2 appeared simultaneously in the second window for $800 \mathrm{msec}$. Following this period, Picture 1 for verifying Sentence 1 appeared in the third window. The subject pressed one of two keys to decide on a "true" or "false" response. Immediately after the subject's response (irrespective of correct or incorrect responses), Picture 1 of the third window disappeared, and Picture 2 for verifying Sentence 2 was presented in the fourth window. The subject pressed one of another set of keys to indicate his "true" or "false" response. The four windows of the tachistoscope were arranged from left to right. The presentation order of the 32 sequences of events was random. For each subject, 8 practice sequences preceded the 32 experimental sequences.

\section{Results and Discussion}

Comprehension time. With respect to the comprehension times of Sentence 1, affirmatives were comprehended faster than negatives. The mean comprehension times for affirmatives and negatives were .85 and $.89 \mathrm{sec}$, respectively. The difference was significant $[\mathrm{t}(28)=4.00]$. (All significance tests in this paper are based on $\mathrm{p}<.05$.)

When the comprehension time of a sentence was measured separately from its verification time in Experiment 1, a negative took $40 \mathrm{msec}$ longer than an affirmative. In a different experimental situation, Just and Carpenter (1976) observed subjects' eye movements throughout a trial. They found that the subjects spent $100 \mathrm{msec}$ longer reading negative than reading affirmative sentences. A problem with using subjects' eye movements is that, even if they fixated on a new item, they could continue processing an old item. It was also possible for subjects to start considering strategies for processing a new item while fixating on an old item. In general, however, the present finding of the relative comprehension times for affirmative and negative sentences agrees with the previous studies.

Verification time of Sentence 1. The mean verification times for TA, FA, TN, and FN of Sentence 1 are listed in Table 1. The erroneous responses $(3.1 \%)$ were discarded in computing the means. Clearly, true 
Table 1

Mean Verification Times of TA, FA, TN, and FN

\begin{tabular}{llrrrr}
\hline & & \multicolumn{4}{c}{ Mean Verification Time (Sec) } \\
\cline { 3 - 6 } & & \multicolumn{1}{c}{ TA } & \multicolumn{1}{c}{ FA } & \multicolumn{1}{c}{ TN } & FN \\
\hline \multirow{2}{*}{ Sentence 1 } & Mean & 1.11 & 1.17 & 1.31 & 1.44 \\
& Percent Errors & 1.3 & 2.6 & 4.7 & 3.9 \\
Sentence 2 & Mean & 1.35 & 1.37 & 1.37 & 1.55 \\
& Percent Errors & 14.7 & 10.3 & 14.2 & 20.3 \\
\hline
\end{tabular}

Note-Even for Sentence 2, TA, FA, TN, and $F N$ were classified on the basis of Sentence 1.

sentences were verified faster than false sentences, and affirmatives were verified faster than negatives. An analysis of variance showed that these two effects were significant $[\mathrm{F}(1,28)=8.35$, MSe $=.033$, and $\mathrm{F}(1,28)=36.02$, MSe $=.043$, respectively]. There was no interaction between type of response and type of sentence $(F<1)$.

When the verification time of a sentence was measured separately from the comprehension time in this experiment, a different pattern of verification times for TA, FA, TN, and FN emerged. Affirmatives were verified faster than negatives, as in the previous studies. However, true sentences were always verified faster than false sentences irrespective of affirmatives or negatives. The frequently obtained pattern of $\mathrm{TA}<\mathrm{FA}<\mathrm{FN}<\mathrm{TN}$ verification times, then, reflects constituent-by-constituent comparisons. This statement is particularly supported by a previous study (Liu \& Liang, 1977), because in one experiment of that study the same Sentence 1 and Picture 1 materials of the present experiment were presented simultaneously for verification, and the TA $<$ FA $<$ FN $<$ TN ordering was obtained.

Since Sentence 1 was quite limited in its varieties, the subjects of the present experiment might have adopted the same constituent comparison strategy as noted in the previous studies (e.g., Liu \& Liang, 1977). Presumably, after a few trials in a typical experiment, the subject adopts a strategy of paying attention only to some critical features of a sentence, without even trying to comprehend the meaning of the sentence. The critical features include such sentence constituents as the property of an object (red or green) and the polarity marker. Thus, when a sentence (The dots aren't red) and a picture are presented for verification, the subject pays attention only to the italicized words (n't red). These features are indeed sufficient for the subject to verify a sentence against a picture and make a correct response. This may give rise to a verification behavior depending on constituent-by-constituent comparisons. This situation is less likely to occur in Experiment 1 on the following grounds. In the present experiment, the subjects were required to process not only Sentence 1, but also Sentence 2 before verifying
Sentence 1 against Picture 1 . In verifying Sentence 1 against Picture 1, the subjects were forced to discriminate between Sentence 1 and Sentence 2. Then, the subject would naturally pay attention to every word of a sentence, since Sentence 1 had no word in common with Sentence 2.

Verification time of Sentence 2 as affected by Sentence 1. In order to see how effectively Sentence 2 had stopped the processing of Sentence 1, the error rate of verifying Sentence 2 would be a good measure. This error rate was found to be $14.9 \%$. It was larger than the error rate of verifying Sentence 1 and lower than $50 \%$, thus satisfying the requirements. It may be reasoned that if Sentence 1 was negative or false, the verification times of Sentence 2 might be affected, since negatives and false sentences were found to take longer verification times. According to an analysis of variance performed on the second row of Table 1 , sentence type and response type of Sentence 1 had significant effects on the verification times of Sentence $2[\mathrm{~F}(1,28)=8.61, \mathrm{MSe}=.037$, and $\mathrm{F}(1,28)=8.44$, MSe $=.031$, respectively]. The Sentence Type by Response Type interaction was not significant $[\mathrm{F}(1,28)=2.31$, MSe $=.082]$.

When Sentence 1 was negative and/or false, it took longer to verify Sentence 2, apparently because either encoding or retrieval of Sentence 2 suffered. Since Sentence 2 was presented when the subject was ready to accept it, the retrieval explanation would be more plausible than the encoding explanation. With respect to the retrieval explanation, there are two possibilities. According to one possibility, a longer verification time of Sentence 1 that is negative and/or false necessarily entails a longer retention interval for Sentence 2. According to another possibility, a heavier processing load accompanies the verification of a negative or false sentence and hence affects retrieval of Sentence 2. It should be noted that, although Sentence 2 verification was necessarily dependent on Sentence 1 verification, the data of Sentence 1 verification were not dependent on the former. This is because the number and type of Sentence 2 were counterbalanced following Sentence 1.

Relationship between comprehension and verification. The correlation between mean comprehension and mean verification times of Sentence 1 was significant $(r=.41)$. This means that when a subject was faster in comprehending Sentence 1, he or she was in general faster in verifying Sentence 1 . Our main interest is also in finding out whether two groups of subjects had different mean comprehension times (Sentence 1) when these two groups were formed on the basis of different patterns of verification times (Sentence 1), as was found in the study of McLeod et al. (1978). The subjects were divided into two groups on the basis of whether their verification 
times of TN and FN had the TN $<$ FN or the FN $<$ TN ordering. There were 15 subjects with the TN $<$ FN ordering and 14 with the $\mathrm{FN}<\mathrm{TN}$ ordering. The former group may be considered poorly fit to the constituent comparison model; the latter are well fit to the model. The mean comprehension times of these two groups were .876 and $.873 \mathrm{sec}$, respectively. This difference was not significant [t $(27)=.04]$. From this observation, it is clear that the two groups of subjects with different TN and FN orderings did not adopt different criteria in comprehending Sentence 1 .

\section{EXPERIMENT 2}

Based on their experimental results, McLeod et al. (1978) reasoned that two distinct models of sentence verification times are required: $A$ general linguistic model is supposed to apply to the well-fit group of subjects, and a general pictorial model predicts behavior of the poorly fit group of subjects. They argued that the pictorial model subjects must execute the additional step of converting a linguistically based proposition into an expected pictorial representation before indicating sentence comprehension. On the other hand, during the verification stage, the pictorial model subjects do not have to convert the initial visual representation of the picture into a proposition, whereas this conversion is required for the linguistic model subjects. Consequently, pictorial model subjects should be faster in the verification stage.

We have presented evidence in Experiment 1 that there was even a positive correlation between the comprehension and verification times of Sentence 1. Had the reasoning of McLeod et al. (1978) applied to the situation of Experiment 1 , a negative correlation between the comprehension and verification times should have been obtained. Moreover, the mean comprehension time of the well-fit group of subjects (defined by the FN $<$ TN ordering) was not different from that of the poorly fit group of subjects (defined by the TN $<$ FN ordering). These results should not be viewed as disconfirmation of the model proposed by McLeod et al. On the other hand, these results only indicate that under a different experimental condition, all subjects could without exception be forced to construct a unitary representation out of the surface elements of a sentence during the comprehension stage:

What is the nature of this unitary representation? According to the TN $<$ FN ordering obtained in Experiment 1, this unitary representation may at least partially be pictorial, since Sentence 1 of Experiment 1 could be falsified in only one way with a consequence of allowing recoding to take place. The purpose of Experiment 2 was to have subjects verify
Sentence 1 that could be falsified in many ways, so that the recoding strategy would not be appropriate.

\section{Method}

Subjects and Materials. The subjects were 17 freshmen of an introductory psychology class. They participated in the experiment to fulfill a course requirement. None of these subjects served in Experiment 1.

Sentence 1 was "These dots are (aren't) black," "These dots are (aren't) white," "These dots are (aren't) red," or "These dots are (aren't) green." Picture 1 was a picture of six black dots, a picture of six white dots, a picture of six red dots, or a picture of six green dots. Sentence 2 was "Triangle lack (not lack) a part" or "Rectangle lack (not lack) a part." Picture 2 was a picture of a triangle lacking an angle, a picture of a complete triangle, a picture of a rectangle lacking an angle, or a picture of a complete rectangle. In the Chinese language Sentence 1 and Sentence 2 had no common word, and "schematic" sentences are legitimate.

Procedure. Except for a difference in sentence and picture materials, the procedure of Experiment 1 was used. For each used as Sentence 1, three pictures (same picture for an affirmative sentence and three different pictures for a negative sentence) that were true of the sentence were used as Picture 1 , and three pictures (three differenc pictures for an affirmative sentence and same picture for a negative sentence) were used as Picture 1 for falsifying the sentence. Thus, each subject received 48 sequences (trials) of Sentence 1, Sentence 2, Picture 1, and Picture 2. Eight practice trials also preceded these experimental trials.

\section{Results and Discussion}

Comprehension time. The mean comprehension times for affirmatives and negatives were computed separately for Sentence 1 with different predicates (Table 2). Although the overall difference in the comprehension times of affirmatives and negatives was in the expected direction, the difference was not significant $(F<1)$. Type of predicate was a significant source of variance $[\mathrm{F}(3,48)=3.60, \mathrm{MSe}=.039]$. The interaction between type of sentence and type of predicate was not significant $(F<1)$.

According to Table 2, the significant effect of predicate was apparently due to the faster comprehension times of Sentence 1 with a "black" predicate. This is understandable, if it is assumed that affirmatives and negatives are not separable in affecting the comprehension times and that the subject constructs a unitary representation in comprehending a sentence. In comprehending the sentence, "These dots aren't black," the subject can more easily attain a unitary representation by aggregating white, red, and green dots to obtain "nonblack dots" (perhaps represented as "bright dots"). In the case of sentences

Table 2

Mean Comprehension Times (in Seconds) of Sentences With Different Predicates

\begin{tabular}{lccccc}
\hline \multirow{2}{*}{$\begin{array}{c}\text { Type of } \\
\text { Sentence }\end{array}$} & Black & White & Red & Green & Mean \\
\cline { 2 - 5 } & -1.34 & 1.40 & 1.41 & 1.36 \\
Affirmative & 1.28 & 1.34 & 1.40 \\
Negative & 1.29 & 1.41 & 1.40 & 1.44 & 1.38 \\
\hline
\end{tabular}


Table 3

Mean Verification Times (in Seconds) of Sentences With Different Predicates

\begin{tabular}{|c|c|c|c|c|c|c|c|c|}
\hline \multirow[b]{3}{*}{ Predicate } & \multicolumn{4}{|c|}{ Affirmative Sentence } & \multicolumn{4}{|c|}{ Negative Sentence } \\
\hline & \multicolumn{2}{|c|}{ True } & \multicolumn{2}{|c|}{ False } & \multicolumn{2}{|c|}{ True } & \multicolumn{2}{|c|}{ False } \\
\hline & Mean & $\begin{array}{c}\text { Percent } \\
\text { Error }\end{array}$ & Mean & $\begin{array}{c}\text { Percent } \\
\text { Error }\end{array}$ & Mean & $\begin{array}{l}\text { Percent } \\
\text { Error }\end{array}$ & Mean & $\begin{array}{r}\text { Percent } \\
\text { Error }\end{array}$ \\
\hline $\begin{array}{l}\text { Black } \\
\text { White } \\
\text { Red } \\
\text { Green }\end{array}$ & $\begin{array}{l}1.20 \\
1.32 \\
1.26 \\
1.33\end{array}$ & $\begin{array}{r}.0 \\
3.9 \\
5.9 \\
5.9\end{array}$ & $\begin{array}{l}1.50 \\
1.76 \\
1.37 \\
1.61\end{array}$ & $\begin{array}{l}3.9 \\
3.9 \\
3.9 \\
2.0\end{array}$ & $\begin{array}{l}1.67 \\
1.72 \\
1.61 \\
1.56\end{array}$ & $\begin{array}{l}7.8 \\
3.9 \\
3.9 \\
9.8\end{array}$ & $\begin{array}{l}1.80 \\
1.77 \\
1.53 \\
1.59\end{array}$ & $\begin{array}{l}3.9 \\
2.0 \\
5.9 \\
7.8\end{array}$ \\
\hline Mean & 1.28 & 3.9 & 156 & 3.4 & 1.64 & 5.9 & 1.67 & 4.9 \\
\hline
\end{tabular}

with "red" and "green" as predicates, this unitary representation is not readily achieved. In the case of a sentence with a "white" predicate, the difficulty level of attaining this unitary representation seems to fall in between the above two extremes.

Verification time of Sentence 1. The mean verification time and the percentage of errors of Sentence 1 under each condition are presented in Table 3. Since the error rate of verifying Sentence 2 was $19.48 \%$, an introduction of Sentence 2 was effective in separating the comprehension and verification processes of Sentence 1. As in Table 1, more errors were generally found in verifying negatives than in verifying affirmatives. An analysis of variance performed on the mean verification times of Sentence 1 showed that type of predicate was not a significant source of variance $[F(3,48)=2.38, \mathrm{MSe}=.194]$. Type of predicate did not significantly interact with type of sentence (affirmative-negative factor) $[\mathrm{F}(3,48)=1.70$, MSe $=.141]$ or with type of response $[F(3,48)=1.03$, MSe $=.179]$. However, affirmatives were verified faster than negatives $[\mathrm{F}(1,16)=10.25$, MSe $=.369]$, "true" responses were faster than "false" responses $[\mathrm{F}(1,16)=18.17, \mathrm{MSe}=.093]$, and type of sentence interacted with type of response $[F(1,16)=6.13$, MSe $=.170]$. This interaction indicates that, although TN tended to be verified faster than FN, this difference was much smaller than the difference found with TA and FA.

Referring to Table 3, since the row effect was not significant, nor its interaction with any of the other variables, it is only necessary to pay attention to the data of the last row. However, there is an important sign that is consistent with the present viewpoint. When a negative sentence (such as one with a "black" predicate) was recodable, the TN $<$ FN ordering tended to prevail (first row of Table 3). Otherwise, TN tended to be nearly identical to FN.

\section{GENERAL DISCUSSION}

The main purpose of the present study was to introduce a masking stimulus (Sentence 2) to stop the further processing of a sentence (Sentence 1) in a sentence-picture verification task, so that the verifica- tion process would not be contaminated by the comprehension process in the verification stage. Within this framework, so that Sentence 1 is not forgotten from short-term memory in the face of processing a masking sentence, it is inferred that the subject necessarily constructs a unitary representation from a presented sentence.

Following a fixed retention interval, the subject's verification of a sentence may be divided into two stages: retrieval of a sentence to be verified and verification of the retrieved sentence against a picture. By the very meaning of unitary representation, the time of retrieving a sentence may be assumed not to depend upon its type. In other words, it is assumed that retrieval times of affirmatives and negatives are nearly equivalent. The pattern of obtained verification times is then solely dependent on what happend during the second stage of verification.

Since TNs were found to be nearly identical to FNs in Experiment 2 when a recoding strategy was generally inapplicable, the TN $<$ FN ordering obtained in Experiment 1 may be attributed to the subjects' recoding of negatives into their affirmative counterparts. There is strong reason to doubt this argument. With the present experimental technique, if such recoding had taken place, the unitary representation constructed for negatives would have not been different from that for affirmatives. Consequently, the verification time of negatives should not be different from that of affirmatives. This contradicts the obtained results. A theoretical model capable of accounting for the present results is presented below.

\section{Theoretical Model}

This model assumes that for those sentences considered in most sentence-picture verification tasks there are three levels of linguistic abstraction (comprehension). Only with the second or third level of comprehension does the subject construct a unitary representation by finding a relationship among all sentence elements. Each level of linguistic abstraction is associated with an adjunct recoding process, which in turn determines how the verification process takes place.

Three levels of linguistic abstraction. At the first 
level of linguistic abstraction, the subject merely picks up the meanings of individual words without abstracting a higher order meaning from a group of words. In some sentence-picture verification tasks, it is necessary only to pick up the meanings of a small number of words to decide whether there is a match or a mismatch between a picture and a sentence. Some examples at this level of linguistic abstraction were presented in the beginning of this paper. In Figure 1 this level corresponds to the lowest level $\left(\mathrm{L}_{1}\right)$ of linguistic abstraction. If a sentence has four words $\left(e_{1}, e_{2}, e_{3}\right.$, and $\left.e_{4}\right)$, this level of abstraction may be identified by a subset (including an improper subset) of words separated by commas [e.g., $\left.\left(\mathrm{e}_{3}, \mathrm{e}_{4}\right)\right]$.

At the second level of linguistic abstraction $\left(\mathrm{L}_{2}\right)$, the subject attains a higher order meaning by combining some but not all words of a sentence. A convenient example is "(not, dots-are-red)," constructed from the sentence, "Dots aren't red." In this example, the subject attains a unitary representation (dots-arered) from three words, but he fails to include the fourth word (not) in this unitary representation. Figure 1 shows this example.

At the third level of linguistic abstraction $\left(\mathrm{L}_{3}\right)$, the subject combines the abstracted representations of $L_{2}$ to form a completely unitary representation of a negative sentence.

With this model it takes longer to comprehend negatives than affirmatives because the former require one additional level of linguistic abstraction. Second, affirmatives are verified faster than negatives because $\mathrm{L}_{3}$ representations for negatives are more abstract than $L_{2}$ representations for affirmatives. The $L_{3}$ representations may be likened to "degraded" stimuli whose comparison with pictures certainly takes a longer time. Third, if it is assumed that the verification time is faster when a sentence representation and a picture match, then the result of the faster verification time for "true" responses than for "false" responses obtained in Experiment 1 can be explained. Although there is a question of how a unitary representation and a picture can be compared, Bever (1975) contends that a common language is not required to compare a picture and a nonpictorial representation.

The explanation of the faster verification time for "true" responses than for "false" responses does not apply to the results of Experiment 2, because the verification time of TN was nearly identical to that of FN, even though TAs were verified faster than FAs. One solution for this difficulty is to assume that unitary representations at $\mathrm{L}_{3}$ differ with respect to whether negative sentences are recodable or unrecodable. It should be recalled that a negative sentence of Experiment 1 was recodable, whereas that of Experiment 2 was unrecodable. A difficulty with this solution is that the same negative sentence can be

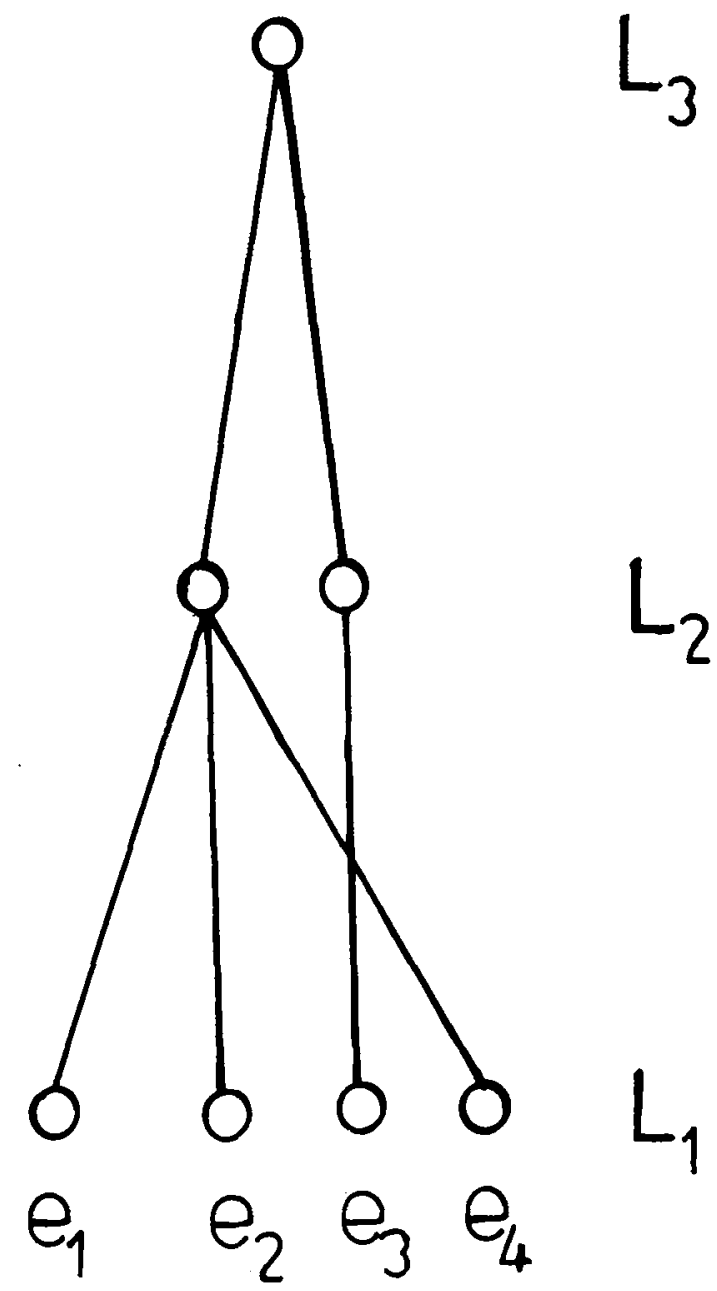

Figure 1. Three levels of linguistic abstraction. At $\mathbf{L}_{2}$ there are two nodes consisting of $\left(e_{1}-e_{2}-e_{4}\right)$ and $e_{3} ;$ at $L_{3}$ there is only a single node, $\left(e_{1}-e_{2}-e_{4}-e_{3}\right)$. In this example, $e_{1}=\operatorname{dots}, e_{2}=$ are, $e_{3}=$ not, and $e_{4}=$ red.

recodable in one situation and unrecodable in another. From the standpoint of analysis, it is desirable to have the linguistic abstraction (unitary representation) dependent only on the sentence without depending on the linguistic environment. Here the linguistic environment refers to a set of sentences to which a given sentence belongs. The composition of a set of sentences determines whether a given negative sentence is recodable or unrecodable. An alternative solution is then to assume that a recoding process takes place in parallel with the linguistic abstraction. This argument naturally leads to consideration of a recoding process as playing an important role in the present model. It should be noted that in asserting negative sentences as unitary, the present model differs from most propositional theories (e.g., Kintsch, 1974).

Adjunct recoding process. The presence of adjunct recoding processes in reading has been noted for a long time (e.g., Bugelski, 1969; Huey, 1908; Paivio, 
1971). Huey (1908) argued essentially that reading for meaning (linguistic abstraction in the present model) involves the arousal of images and feelings by sentences. Paivio and Begg's (Note 1) subjects pressed a button to expose sentences one at a time and released it as soon as they had an image or understood the sentence. Paivio and Begg found that both concrete and abstract sentences took longer to arouse images (adjunct recoding process) than to be understood (linguistic abstraction) and that abstract sentences took longer to be understood and to arouse images than did concrete sentences. In reading easy materials, the adjunct recoding process plays a less important role because a person reads the following sentences before imageries of the current sentences are aroused.

There is still a dispute as to whether these adjunct recoding processes are images or merely propositional. For the present purpose both terminologies are used to aid exposition of the present model.

When a sentence is encoded, the principal linguistic abstraction is naturally accompanied with the adjunct recoding process. If a sentence is encoded for a later verification test with a picture, the adjunct recoding process will be adjusted to take features more in common with pictures. It is assumed that recodability of a sentence is not all or none but forms a continuum. The adjunct recoding process for a given set of sentences that includes fewer recodable negative sentences takes a longer time for its completion, resulting in a longer comprehension time. This explains why the mean comprehension times of Experiment 2 were without exception longer than those of Experiment 1. Notice that both principal linguistic abstraction and adjunct recoding process are integral parts of the same comprehension process in the present situation.

For less recodable negative sentences, the resultant adjunct recoding process will be more abstract and have fewer features in common with pictures. In this case, FN will be verified nearly as fast as TN because a match or mismatch between the sentence and picture representations is not obvious and depends on inferences. This explains the pattern of verification times obtained in Experiment 2. For clearly recodable negative sentences, the resultant adjunct recoaing process will have more features in common with pictures. In the verification stage, a match between the sentence and picture representations can easily be determined. The subject presses the "false" key only when the first test of a sentence-picture match fails, hence consuming extra time. This explains the TN $<$ FN ordering of Experiment 1.

Relation with previous results. With the present theoretical scheme, it is possible to view many previous results in a proper perspective. Two key questions are: to which level of linguistic abstraction did the subjects reach in their comprehension of sentences, and how much were the subjects induced to adopt the strategy of relying upon the adjunct recoding process for a later verification test?

If sentences were comprehended at $\mathrm{L}_{2}$ or $\mathrm{L}_{1}$, the pattern of verification times, TA $<$ FA $<$ FN $<$ TN, predicted by the constituent comparison models should be obtained (e.g., Carpenter \& Just, 1975; Clark \& Chase, 1972; Gough, 1965). Second, Glushko and Cooper (1978) correctly observed that, as subjects have more time to prepare for the test figure, "true" (or "same") responses were faster than "false" (or "different") responses. Within the present theoretical framework, if subjects had more time to prepare for the test figure, the adjunct recoding process would be aroused intentionally to be ready for comparison with a picture. In this situation, it has been noted that "true" responses tend to be faster than "false" responses. Third, Glushko and Cooper also found that the preparation and verification times are not manifestations of independent processes when the data of the simultaneous and subject-controlled conditions are cross-examined. According to the present view, the subjects tend to reach $L_{1}$ or at most $L_{2}$ in their linguistic abstraction under the simultaneous condition, but they go further under the subjectcontrolled situation. In this way the modes of comprehension and verification will be different under the two conditions. This naturally argues against the independence of the comprehension and verification processes in the situations under consideration.

More important, speech recoding may be regarded as one form of the adjunct recoding process. Its role in reading in particular and in short-term memory in general has attracted the attention of many investigators.

Although the present model is similar to a levelsof-processing approach (Craik \& Lockhart, 1972) in appearance, there is an important difference. While the former is particularly suitable to those situations in which subjects can extract visual and phonological features without carrying out semantic processing, the latter is constructed for usual situations in which semantic processing goes in conjunction with visual and phonological processing. It is further assumed that semantic and visual-phonological processing are integral parts of the same comprehension process.

\section{REFERENCE NOTE}

1. Paivio, A., \& Begg, I. Imagery and comprehension latencies as a function of sentence concreteness and structure (Research Bulletin 154). London, Ontario: University of Western Ontario, Department of Psychology, 1970.

\section{REFERENCES}

Bever, T. G. Some problems that arise if we insist on distinguishing language and thought. In D. Aaronson \& R. Rieber (Eds.), 
Developmental psycholinguistics and communication disorders. Annals of the New York Academy of Sciences, 1975, 263, 76-86.

Bransford, J. D., Barclay, J. R., \& Franks, J. J. Sentence memory: A constructive versus interpretive approach. Cognitive Psychology, 1972, 3, 193-209.

BUGELSKI, B. R. Learning theory and the reading process. In The 23rd annual reading conference. Pittsburgh: University of Pittsburgh Press, 1969.

Carpenter, P., \& Just, M. A. Sentence comprehension: A psycholinguistic model of sentence verification. Psychological Review, 1975, 82, 45-73.

Clark, H. H., \& Chase, W. G. On the process of comparing sentences against pictures. Cognitive Psychology, 1972, 3, 472-517.

Craik, F. I. M., \& Lockhart, R. S. Levels of processing: A framework for memory research. Journal of Verbal Learning and Verbal Behavior, 1972, 11, 671-684.

Fillenbaum, S. Memory for gist: Some relevant variables. Language and Speech, 1966, 9, 217-227.

Glushko, R. J., \& Cooper, L. A. Spatial comprehension and comparison processes in verification tasks. Cognitive Psychology, 1978, 10, 391-421.

Gough, P. B. Grammatical transformations and speed of understanding. Journal of Verbal Learning and Verbal Behavior, $1965,5,107-111$.

Gough, P. B. The verification of sentences: The effects of delay of evidence and sentence length. Journal of Verbal Learning and Verbal Behavior, 1966, 5, 492-496.
HUEY, E. B. The psychology of pedagogy of reading. New York: Macmillan, 1908.

Just, M. A., \& CARpenter, P. A. Comprehension of negation with quantification. Journal of Verbal Learning and Verbal Behavior, 1971, 10, 244-253.

Just, M. A., \& Carpenter, P. A. Eye fixations and cognitive processes. Cognitive Psychology, 1976, 8, 441-480.

$\mathrm{KINTSCH}, \mathrm{W}$. The representation of meaning in memory. Hillsdale, N.J: Erlbaum, 1974.

Liang, K., \& LiU, I. Process of verifying multi-attribute sentences against pictures. Acta Psychologica Taiwanica, 1977, $19,39-47$.

LIU, I., \& LIANG, K. Verifying affirmative and negative sentences. National Science Council Monthly, 1977, 5, 432-441.

McLeod, C. M., Hunt, E. B., \& Mathews, N. N. Individual differences in the verification of sentence-picture relationship. Journal of Verbal Learning and Verbal Behavior, 1978, 17, 493-507.

Paivio, A. Imagery and verbal processes. New York: Holt, Rinehart, \& Winston, 1971.

SACHS, J. Recognition memory for syntactic and semantic aspects of connected discourse. Perception \& Psychophysics, 1967, 2, 437-442.

Trabasso, T., Rollins, H., \& Shaughnessy, E. Storage and verification stages in processing concepts. Cognitive Psychology, 1971, 2, 239-289.

(Received for publication August 9, 1979; revision accepted October 31,1979 .) 